AperTO - Archivio Istituzionale Open Access dell'Università di Torino

\title{
Antiphospholipid syndrome
}

\section{This is the author's manuscript}

Original Citation:

Availability:

This version is available http://hdl.handle.net/2318/1609788

since 2019-11-14T12:22:02Z

Published version:

DOI:10.1016/j.berh.2016.04.002

Terms of use:

Open Access

Anyone can freely access the full text of works made available as "Open Access". Works made available under a Creative Commons license can be used according to the terms and conditions of said license. Use of all other works requires consent of the right holder (author or publisher) if not exempted from copyright protection by the applicable law. 
This Accepted Author Manuscript (AAM) is copyrighted and published by Elsevier. It is posted here by agreement between Elsevier and the University of Turin. Changes resulting from the publishing process - such as editing, corrections, structural formatting, and other quality control mechanisms - may not be reflected in this version of the text. The definitive version of the text was subsequently published in BAILLIERE'S BEST PRACTICE \& RESEARCH: CLINICAL RHEUMATOLOGY, 30 (1), 2016, 10.1016/j.berh.2016.04.002.

You may download, copy and otherwise use the AAM for non-commercial purposes provided that your license is limited by the following restrictions:

(1) You may use this AAM for non-commercial purposes only under the terms of the CC-BY-NC-ND license.

(2) The integrity of the work and identification of the author, copyright owner, and publisher must be preserved in any copy.

(3) You must attribute this AAM in the following format: Creative Commons BY-NC-ND license (http://creativecommons.org/licenses/by-nc-nd/4.0/deed.en), 10.1016/j.berh.2016.04.002

The publisher's version is available at:

http://linkinghub.elsevier.com/retrieve/pii/S152169421630002X

When citing, please refer to the published version.

Link to this full text:

http://hdl.handle.net/ 


\title{
Antiphospholipid syndrome.
}

Khamashta $\mathrm{M}^{1}$, Taraborelli $\mathrm{M}^{2}$, Sciascia $\mathrm{S}^{3}$, Tincani $\mathrm{A}^{4}$.

'Lupus Research Unit, The Rayne Institute, Division of Women's Health, Kings College London, St. Thomas Hospital, London, United Kingdom; Department of Rheumatology, Dubai Hospital, Dubai, United Arab Emirates. Electronic address: munther.khamashta@kcl.ac.uk.

${ }^{2}$ Rheumatology and Clinical Immunology Unit, Spedali Civili of Brescia, Piazzale Spedali Civili of Brescia 1, 25123 Brescia, Italy. Electronic address: mara.taraborelli@gmail.com.

${ }^{3}$ Center of Research of Immunopathology and Rare Diseases - Coordinating Center of Piemonte and Valle d'Aosta Network for Rare Diseases, S. Giovanni Bosco Hospital, University of Turin, Piazza del Donatore di Sangue, 3, 10154 Turin, Italy. Electronic address: savino.sciascia@unito.it.

${ }^{4}$ Rheumatology and Clinical Immunology Unit, Spedali Civili and University of Brescia, Piazzale Spedali Civili of Brescia, 1, 25123 Brescia, Italy. Electronic address: angela.tincani@gmail.com.

\begin{abstract}
Antiphospholipid syndrome (APS) is an autoimmune condition characterized by the occurrence of thrombosis (arterial and/or venous), often multiple, and/or pregnancy morbidity. Thrombosis is one of the major disease mechanisms, mainly caused by activating endothelial cells, monocytes, and platelets. At present, the management of APS patients with a history of thrombosis is based on long-term antithrombotic therapy, due to the high rate of recurrent thrombosis (29\% per year without treatment). Obstetrical APS includes heterogeneous pregnancy complications whose pathogenesis has been increasingly elucidated in the past years. This is due to the current management and treatment, as $80 \%$ of APS patients achieve a live birth. The standard approach of APS is not supported by extensive evidence and the best options for refractory and incomplete cases need to be clarified. New and promising molecules are under investigation.
\end{abstract}

\section{KEYWORDS:}

Antiphospholipid antibodies; Antiphospholipid syndrome; Pregnancy; Thrombosis; $\beta 2$ glycoprotein-I

\section{Introduction}

Antiphospholipid syndrome (APS) is an autoimmune condition characterized by the occurrence of thrombosis (arterial and/or venous), often multiple, and/or morbidity in pregnancy (recurrent miscarriages, fetal deaths (FDs), and late pregnancy complications such as preeclampsia (PreE) and intrauterine growth restriction (IUGR)), in the presence of antiphospholipid antibodies (aPLs), typically the antibodies included in the classification criteria for APS, lupus anticoagulant (LA), anticardiolipin (aCL), or anti- $\beta 2$ glycoprotein-I (anti- $\beta 2 \mathrm{GPI}$ ) antibodies (Table 1), although other "non-criteria" antibodies may also play a role. APS may be associated with other autoimmune diseases, mainly systemic lupus erythematosus (SLE), but it can also be seen in patients having no 
other definable rheumatologic condition (primary APS). Occasionally, it can accompany other conditions, such as infections, drugs, or malignancies [1].

In this review, we discuss recent advances in the diagnosis of APS including new insights into the pathogenesis and the implications for the identification of new biomarkers. In addition, the importance of recent longitudinal observational studies in understanding the natural history of the syndrome and for embedding research in clinical practice will be elucidated.

\section{Thrombotic APS}

\section{Pathogenesis}

Although the full pathogenesis of APS is not yet clear, the key mechanisms have been described recently. Thrombosis is one of the major disease features, driven by multiple mechanisms including activation of endothelial cells, monocytes, platelets, coagulation, and complement pathways in addition to inhibition of fibrinolytic and anticoagulation pathways [2]. Recent evidence indicates that vasculopathy, enhanced mainly by severe intimal hyperplasia, can also play a role in arterial vascular occlusion (due to stenotic lesions) and pregnancy morbidity [3]. In support of this hypothesis, Canaud et al. [3] recently demonstrated that the vascular endothelium of proliferating intrarenal vessels from patients with APS nephropathy showed indications of activation of the mammalian target of rapamycin (mTOR) pathway. In cultured vascular endothelial cells, IgG antibodies in patients with APS stimulated mTOR through the phosphatidylinositol 3-kinase (PI3K)-AKT pathway. Patients with APS nephropathy who required renal transplantation and were treated with sirolimus (also known as rapamycin, which can inhibit mTOR) showed no recurrence of vascular lesions and decreased vascular proliferation on biopsy compared with patients with aPLs who were not treated with sirolimus. Of 10 patients treated with sirolimus, seven $(70 \%)$ had a functioning renal allograft 144 months after transplantation versus three of 27 untreated patients (11\%). This study conducted in patients with primary and secondary APS nephropathy, which is mainly mediated by vasculopathy rather than thrombosis, revealed that the activation of the mTOR enzyme stimulates intimal hyperplasia, leading to the formation of the chronic vascular lesions seen in APS. Confirmatory studies and more detailed investigation of the steps leading to mTOR pathway recruitment and the molecular consequences of its activation are still needed. However, there is growing evidence that these enzymes can also induce a prothrombogenic phenotype leading to thrombosis [4]. This might represent an obstacle in proceeding further to test drugs such as sirolimus in patients with APS [4].

\section{Diagnosis and thrombotic risk assessment}

\section{aPL profile and thrombotic risk assessment}

The relationship between aPLs and thrombosis has been widely investigated, and a strong association between aPLs and thrombotic events has been confirmed [5]. However, the strength of these results from clinical studies is limited by factors such as differences in the study design and eligibility criteria, 
and by the diversity of aPLs in terms of types, isotypes, cutoff, and laboratory methods used for their detection.

In clinical practice, it is common to question whether patients with similar clinical manifestations but different patterns and/or combinations of positive aPL test results should be considered equivalent. Patients may have single, double, or triple aPL positivity; have single or multiple isotypes of aCL and anti- $\beta 2 \mathrm{GPI}$; or show low versus high titers of antibodies. Whether heterogeneity of "aPL profiles" represents a spectrum of different categories in terms of prognosis and treatment is still a matter of debate.

Recent studies [6] and [7] suggest that multiple positive tests for aPLs are more frequently associated with thromboembolic events than a single positive test. However, the presence of a single positive test still has to be considered when assessing thrombotic risk, especially in the context of concomitant autoimmune disease or other cardiovascular risk factors [8].

Available studies suggest that LA is the single aPL most strongly related to thrombosis [5]. Data from a systematic review of $>7000$ patients and controls from 25 studies showed that LA has an odds ratio (OR) for thrombosis 5-16 times higher than controls. By contrast, isolated positivity for other aPLs was weakly associated with clinical manifestations of APS [9] and [10]. Although LA positivity increased the risk of stroke 48 -fold and the risk of myocardial infarction 11 -fold, anti- $\beta 2 \mathrm{GPI}$ only doubled the risk of stroke, with no effect on myocardial infarction [10]. The role of aCL in the absence of LA was also analyzed, and no associated increased risk for stroke or myocardial infarction was reported [10].

By contrast, some observational studies suggest that patients with SLE and isolated medium-high titer, and persistently positive aCL (defined as more than two-third of positive serial determinations), had an increased risk of thrombosis, while those with occasional aCL positivity did not [11].

Combined aPL positivity has been associated with an increased thrombotic risk [6]. The so-called triple-positive population ( $\mathrm{LA}+\mathrm{aCL}+$ anti- $\beta 2 \mathrm{GPI}$ ) has been postulated to be the highest risk group [7]. Recently, our group evaluated several combinations of aPL specificities in an attempt to determine the profile that provides the best diagnostic accuracy, not only for APS as a whole but also for thrombosis and pregnancy morbidity independently [12]. Testing for six aPLs resulted in 23 possible combinations. The profile of LA + anti- $\beta 2 \mathrm{GPI}+$ anti-phosphatidylserine-prothrombin, aPS/PT, had the best diagnostic accuracy for APS as a whole and individually for both thrombosis and/or pregnancy loss (OR 3.73 (95\% confidence interval (CI) 1.82-5.38) and OR 3.75 (95\% CI 2.136.62), and/or OR 4.82 (95\% CI 2.17-10.72), respectively), and the best specificity when compared with all the other possible combinations [12]. Multiple aPL positivity also seems to play a role in terms of antibody persistence, and individuals testing positive for more than one aPL tend to have more stable antibody levels on repeated determinations[13].

In a pediatric population, a statistically significant association between first thromboembolism and persistently positive aPLs, with an overall sixfold increase in the risk for thrombosis, was also reported. These data suggest that the detection of persistent aPLs is clinically meaningful in children with, or at risk of, thromboembolism [14]. 
The clinical value of different aPL profiles when assessing the risk of recurrence is also a matter of debate. A recent meta-analysis by Garcia et al. [15] showed that patients with first venous thromboembolism (VTE) who have aPLs have a higher risk of recurrent VTE when compared with patients without aPLs [15]. However, testing for aPLs varied with respect to the antibody sought (aCL, anti- $\beta 2$ GPI, and/or LA), with some studies testing for one aPL, and others testing for more than one, limiting the conclusions drawn.

The aPL risk categories according to profile are shown in Table 2.

\section{Non-criteria aPLs}

It has been proposed that several autoantibodies besides aCL, LA, and anti- $\beta 2$ GPI are relevant to APS. These antibodies are directed against other plasma proteins from the coagulation cascade (i.e., PT and/or PS-PT complexes), to specific domains of $\beta 2$ GPI, or interfere with the anticoagulant activity of annexin A5 (A5) [16]. The clinical utility of these newly developed assays and their clinical value in assessing thrombotic risk are being investigated.

\section{IgA isotypes}

The issue of the value of immunoglobulin A (IgA) aPL and whether it should be part of the routine diagnostic algorithm has been a subject of intense debate. The current laboratory criteria for APS omit the use of $\operatorname{IgA}$ isotypes for both $\mathrm{aCL}$ and anti- $\beta 2 \mathrm{GPI}$ tests. Some available data support testing for IgA aPLs. As data are based on retrospective studies, case reports, and case series, clear-cut recommendations are difficult to make. In addition, comparison between these studies is difficult due to differences in design, population studied, the non-standardized assays used, and the different cutoff chosen for the definition of positivity. As a result and in the absence of well-designed prospective studies, the usefulness of IgA aPL testing in assessing the thrombotic risk in APS continues to be debatable.

Several studies failed to prove the usefulness of IgA aCL and IgA anti- $\beta 2$ GPI testing, because of low prevalence of these antibodies, because they are found along with other aPLs in most cases, and, mainly, because of failure to enhance the diagnostic accuracy of routine testing [17]. Recent studies [18] suggested that isolated IgA anti- $\beta 2$ GPI may identify additional patients with clinical features of APS, and hence recommended testing for these antibodies when other aPLs are negative and APS is suspected. It is important to note that of the 5892 samples tested in this study, only 57 $(<1 \%)$ were exclusively positive for IgA anti- $\beta 2 \mathrm{GPI}$, restricting the application of these recommendations to a limited population of patients.

Based on the published evidence, IgA aPL testing should only be considered for thrombotic risk assessment in selected cases, in the presence of clinical signs and symptoms of APS, mainly associated with SLE, and, particularly, when other aPL tests are negative [19]. 


\section{Antiprothrombin antibodies}

Antiprothrombin antibodies have been proposed as potential new biomarkers for thrombosis and/or pregnancy morbidity in the setting of APS. Antiprothrombin antibodies are commonly detected by enzyme-linked immunosorbent assay (ELISA), using prothrombin coated onto irradiated plates (aPT), or prothrombin in complex with phosphatidylserine (aPS/PT), as antigen. Although these antibodies can coexist in the same patient, aPT and aPS/PT seem to belong to different populations of autoantibodies[20].

The clinical value of antiprothrombin antibodies, detected as either aPT or aPS/PT, has been evaluated with contradictory results [21] and [22]. Most of the studies support an association between antibodies directed to prothrombin, particularly aPS/PT, and clinical manifestations of APS. A recent systematic review based on data from $>7000$ patients and controls suggests that while both aPT and aPS/PT increased the risk of thrombosis, aPS/PT seems to represent a stronger risk factor for both arterial and/or venous thrombosis than aPT [23]. The possibility of antiprothrombin antibodies, particularly aPS/PT, being an additional tool for risk stratification is being actively debated, especially when trying to improve the identification of APS patients negative for the criteria aPLs.

\section{Autoantibodies to domain 1 of $\beta 2 G P I$}

Several studies have investigated the epitope distribution of anti- $\beta 2$ GPI antibodies with the aim of identifying the pathogenic specificities [24] and [25]. The $\beta 2$ GPI molecule has five homologous domains (D): D1-D5. Most of the antibodies have been reported to bind to epitopes located in the domains $\beta 2$ GPI-D1, D4, and D5 and these antibodies may have different clinical interpretations [26]. The main epitope associated with APS has been reported to be cryptic and a conformation-dependent structure that involves different regions of D1 [27]. In an international, multicenter evaluation, an association between anti- $\beta 2$ GPI-D1 antibodies and history of (mostly venous) thrombosis was found [28]. Recent studies have demonstrated that patients with multiple positive test results, usually considered at a higher risk of developing clinical complications, tend to have higher prevalence and higher titers of anti- 32 GPI-D1 antibodies [29]. The hypothesis of antibodies specifically binding the domain I of the $\beta 2$ GPI molecule, as opposed to the whole molecule, as a promising biomarker with a better diagnostic accuracy when compared with current assays for anti- $\beta 2$ GPI is scientifically proven, although it needs to be evaluated further.

\section{Risk factors other than aPLs}

Recently, the role of vascular risk factors in the development of clinical events in patients with APS has been established. Patients with aPLs presenting with thrombosis frequently have one or more additional cardiovascular risk factors such as hypertension, smoking, hypercholesterolemia, or estrogen use [30].

When focusing on arterial events, Matyja-Bednarczyk et al. recently showed that livedo reticularis, as well as hypertension and hypercholesterolemia, increased the risk of arterial thrombosis in APS [31]. Moreover, the interaction between aPLs and smoking and oral contraceptives has been elucidated in a 
case-control study by Urbanus et al. [10] The authors showed that the risk of stroke doubled among LA-positive women who smoked, as compared with nonsmokers, and the risk of stroke among oral contraceptive users multiplied more than sevenfold. All LA-positive women who suffered a myocardial infarction were also smokers.

Coexistent SLE may also have an impact on the risk of thrombosis as SLE is a risk factor for thrombosis per se. Patients with SLE have a higher-than-expected incidence of vascular events, which are not explained completely by traditional vascular risk factors[32]. The combination of SLE and aPLs is particularly a matter of concern, because aPL positivity has been shown to increase the risk of thrombosis in patients with SLE and a diagnosis of SLE appears to further enhance the likelihood of vascular events in patients with aPLs. Indeed, in aPL-positive SLE patients, the annual risk of first thrombosis is higher than in healthy aPL-positive subjects without other cardiovascular risks (4\% vs. $<1 \%)$ [33].

Observational studies suggest that manifestations of APS other than those included in the clinical classification criteria, such as heart valve lesions [11], livedo reticularis, and thrombocytopenia [30] may be associated with thrombosis; however, these associations are not considered strong enough to guide clinical decisions.

\section{The global APS score and mathematical approaches in risk stratification}

One of the unsolved questions is why some aPL carriers never develop any APS manifestation, some develop thrombosis while others present with morbidity during pregnancy and a small number of individuals develop a catastrophic form of APS. Therefore, assessing the risk of developing APS manifestations for an individual with aPLs is very important for physicians [37].

Three score models have been proposed to quantify the risk of thrombosis/obstetric events in APS [8], [38] and [39]. The main aim of these scores is to help clinicians stratify patients according to their risk, identifying those who have a higher likelihood of developing new events and therefore likely to benefit from preventive approaches. The first two scores [38] and [39] focused mainly on the aPL profile, while the most recent one, the Global APS Score or GAPSS [8], also included other variables such as cardiovascular risk factors and autoimmune profile at the time of implementation of the risk model (Table 3).

This score has been internally and externally validated in different large prospective studies of patients with primary and secondary APS [40], [41] and [42]. GAPSS appears to be a promising tool in quantifying the risk of thrombosis and obstetric morbidity in patients with APS.

\section{Treatment}

\section{Primary thromboprophylaxis}

It is still an open question whether prophylactic treatment is needed in subjects with aPLs who have no history of thrombosis. The net benefit of active therapy against placebo has never been clearly proven. However, we recommend a careful thrombotic risk assessment and general measures to control cardiovascular risk factors for all patients with aPLs as part of good clinical practice [43]. The 
avoidance of smoking and control of body weight, blood pressure, and lipids should be considered a primary management goal in all subjects with aPLs [8]. Estrogen-containing oral contraceptive pills or estrogen replacement therapy should be avoided due to their prothrombotic effect.

Autoimmune conditions, mainly SLE, constitute an additional risk factor for thrombosis. Thus, primary thromboprophylaxis should be considered with LDA (75-100 mg/day) in all patients with an underling systemic autoimmune condition and persistent aPLs at medium-high titers (IgM or IgG phospholipid units $>40$ IgG phospholipid units (GPL) or IgM antiphospholipid units (MPL) or $>99$ th percentile). In patients with SLE and with persistently positive aPLs, primary thromboprophylaxis including LDA and/or hydroxychloroquine (HCQ; 200-400 mg/day) is strongly recommended [43]. This is based on studies that have shown that HCQ protects against thrombosis in patients with SLE, including those with aPLs [11]. Although no study has specifically investigated whether the addition of anti-platelet agents offers additional protection, LDA is generally considered effective for primary thromboprophylaxis [43]. Thus, given the recommendation of HCQ therapy in all patients with SLE, the decision to add LDA should be determined on an individual basis. Specifically, the addition of LDA may be appropriate in selected cases, such as those with a high-risk aPL profile (e.g., triple positivity for LA, aCL, and anti- $\beta 2 \mathrm{GPI}$, and/or other concomitant cardiovascular risk factors, and for SLE patients with a history of obstetric APS).

However, while the Physicians' Health Study demonstrated no protection against deep venous thrombosis in men with aCL receiving LDA [44], more recent evidence suggests a protective role of LDA in venous thrombosis, at least in the general population [45].

In asymptomatic carriers of aPLs without an underlying connective tissue disease, the decision regarding thromboprophylaxis should be based on the aPL profile. LDA is suggested for those with a high-risk profile, such as patients with LA, and particularly for triple-positive individuals [41].

Recently, a prospective, multicenter, randomized, open, controlled trial conducted in patients positive for aPLs aimed to examine the efficacy and safety of LDA versus LDA plus low-intensity warfarin in the primary prevention of thrombosis in aPL-positive patients with SLE and/or obstetric morbidity [46]. No differences were observed in the number of thromboses between patients treated with LDA versus those treated with LDA + low-intensity warfarin. In the LDA + warfarin group, more episodes of bleeding were detected. The authors concluded that the LDA + warfarin regimen was significantly less safe than LDA alone.

\section{Prevention of recurrent thrombosis}

At present, the management of APS patients with a history of thrombosis is based on long-term antithrombotic therapy, due to the high rate of recurrent thrombosis $(29 \%$ per year without treatment) [41]. Whether patients with APS should receive the same therapy as the general population with similar manifestations and whether arterial and venous events should be treated differently remain to be answered.

Two randomized, controlled trials have compared high (target international normalized ratio, INR, 3.0-4.0) with standard (target INR 2.0-3.0) intensity of anticoagulation for secondary thromboprophylaxis in patients with APS [47] and [48]. In these trials, no significant differences in 
terms of efficacy or safety between the regimens were observed. However, both suffered from bias due to overrepresentation of patients with their first VTE. Thus, we recommend indefinite anticoagulant therapy with a vitamin K antagonist (VKA) to a target INR of 2.0-3.0 for patients with APS and the first venous event. A reduction in the duration of treatment with VKA can be considered only in patients with a low-risk aPL profile and clear provoking factors (e.g., surgery and prolonged immobilization) at the time of the thrombosis.

Instead, management of arterial events is more controversial and debate persists [49]. The APS and Stroke Study concluded that patients with stroke and aPL not fulfilling classification criteria should be best treated as the general population, with low-dose aspirin [50]. However, at present we use a more aggressive approach for patients with definite APS with arterial disease and/or recurrent events, which might include VKA with a target INR of 3.0-4.0. Occasionally, combined anticoagulant-antiaggregant therapy may also be considered [41]. In fact, recurrences are very infrequent $(0.016-0.031$ events per patient per year) among patients receiving effective oral anticoagulation targeting an INR of $3.0-$ 4.0 [51]. However, the physician has to be aware that high-intensity oral anticoagulation therapy carries a risk of serious hemorrhage, although this risk does not appear higher than that observed in other thrombotic conditions warranting oral anticoagulation [52].

However, the management of VTE is changing rapidly as the new direct oral anticoagulants (DOACs) have been shown to be effective in the management of VTE and they do not require laboratory monitoring [53]. A direct thrombin inhibitor (dabigatran etexilate) and direct anti-Xa inhibitors (rivaroxaban, apixaban, and edoxaban) are currently available. The use of these agents may represent a major step forward as, unlike VKA, they have few reported drug interactions and they do not interact with food or alcohol intake, thereby resulting in more stable anticoagulant intensity. Early evidence for the use of DOACs for secondary thromboprophylaxis for APS patients with a history of VTE is promising, but until data from ongoing clinical trials are available [53], there is not enough evidence to use DOACs in patients with APS and a history of arterial events. However, it is worth noting that LA testing in patients receiving DOACs may be unreliable [53].

\section{Alternative therapies for refractory and difficult cases}

Long-term management of APS patients with recurrent thrombosis may be complicated by fluctuating INR levels, major bleeding, or a high risk of major bleeding. For these reasons, further therapeutic options other than VKA might be considered in selected cases.

Long-term low-molecular-weight heparin (LMWH; e.g., subcutaneous enoxaparin $1 \mathrm{mg} / \mathrm{kg}$ every $12 \mathrm{~h}$ or $1.5 \mathrm{mg} / \mathrm{kg} /$ day or subcutaneous dalteparin $100 \mathrm{IU} / \mathrm{kg}$ every $12 \mathrm{~h}$ or $200 \mathrm{IU} / \mathrm{kg} /$ day), HCQ (200$400 \mathrm{mg} /$ day), or statins have been suggested in these selected cases [54].

Recently, rituximab, an anti-CD20 monoclonal antibody, has been shown to be effective in lifethreatening catastrophic APS, although it has been investigated in a small number of cases [54]. B-cell depletion with rituximab may also be used successfully in patients with aPLs and autoimmunemediated thrombocytopenia and hemolytic anemia [54]. A pilot open-label phase II trial of B-cell depletion with rituximab for non-criteria manifestations of APS concluded that rituximab may 
represent a safe option in APS especially in the case of thrombocytopenia or skin ulcers, although it may not be effective for all non-criteria manifestations (e.g., cardiac valve disease and aPL nephropathy) [55].

There is limited evidence for the use of steroids, other immunosuppressive drugs, or intravenous immunoglobulin (IVIg) in the treatment of APS patients with thrombosis [54]. These drugs should be considered only as rescue therapy in patients with repeated episodes of thrombosis despite adequate anticoagulant therapy, or in catastrophic APS. Anecdotal cases of the use of eculizumab, which targets the complement protein $\mathrm{C} 5$, have also been reported [54].

Other options can be considered on an individual basis, such as the use of intra-arterial fibrinolysis in patients with acute myocardial infarction associated with APS or prostacyclin analogs in patients with severe ischemic necrotic toes associated with APS.

Newer therapeutic agents targeting pathways involved in the development of aPL-mediated clinical manifestations are under investigation. These include blocking of aPL/ $\beta 2$ GPI receptors on target cells, complement and nuclear factor- $\kappa \mathrm{B}$, and P38 mitogen-activated kinase inhibitors. However, the multifactorial mechanisms underlying thrombosis and pregnancy morbidity in APS are still not fully understood and this might limit the development of new targeted therapies for APS. Potentially, the current "antithrombotic" approach to APS patients will be replaced in the future by an "immunomodulatory" approach as our understanding of the mechanisms of aPL-mediated clinical manifestations improves [55].

\section{Obstetrical APS}

\section{Pathogenesis}

The pathogenic role of aPLs in obstetrical APS (OAPS) was initially demonstrated by experimental models showing that the passive transfer of IgG isotype aPLs could induce FD and IUGR in pregnant mice [56]. Three mechanisms for aPL-induced pregnancy morbidity have been postulated: intraplacental thrombosis, defective placentation, and inflammation.

Intraplacental thrombosis and the subsequent alteration of maternal-fetal blood exchanges were thought to be the main pathogenic mechanism of OAPS for many years. This was based on the frequent observation of thrombosis and infarction in histological studies of placentas from APS patients and in vitro studies, suggesting that anti-32GPI antibodies displace the anticoagulant A5 from the trophoblast and endothelial cell monolayers [57]. Nevertheless, placentas from APS patients do not always show evidence of thrombosis and also display inflammatory changes [58]. Moreover, intraplacental thrombosis is not likely to cause early pregnancy losses, based on the fact that significant maternal blood flow is not present in the intervillous space until the end of the first trimester[59].

Based on the ability of anti- $\beta 2$ GPI to react with both the fetal and the maternal sides of the placenta in vitro, a role for aPLs in inducing defective placentation has emerged [60]. In particular, aPLs have been demonstrated to induce direct placental damage by inhibiting trophoblast differentiation and syncytialization, inducing trophoblast apoptosis, impairing trophoblast invasiveness, affecting 
trophoblast expression of adhesion molecules that regulate its adhesion to and invasion of the maternal tissue, and by inhibiting production of angiogenic factor by trophoblasts [59]. The internalization of aPLs by trophoblasts with the subsequent acceleration of cell death and release of debris that can activate maternal endothelial cells has been proposed as an additional mechanism for PreE [61]. Inflammation has been described as one of the main mechanisms of aPL-induced pregnancy morbidity based on (a) the histological demonstration of complement deposition, neutrophil infiltration, and tumor necrosis factor $\alpha$ secretion in decidual tissue; (b) the observation that complement deficiency in animal models or complement inhibition in vivo are protective against obstetrical complications; (c) the evidence of a protective effect of heparin linked to its anticomplement activity; and (d) the observation in in vitro studies that aPLs can induce trophoblasts to produce interleukin- $1 \beta$ by inflammasome activation $*[62]$ and [63].

Although the pathogenic mechanisms of pregnancy morbidity have been increasingly proposed in the past years, research efforts to identify the aPL targets on cell membranes and the intracellular signaling pathways are still ongoing [62]. Heparan sulfate, Toll-like receptors 2 and 4, apolipoprotein E receptor 2 , and annexin II that are expressed by trophoblasts and decidual cells are the main candidate receptors for $\beta 2 \mathrm{GPI}$ identified by in vitro studies [62]. In particular, apolipoprotein E receptor 2 has been suggested to be the key molecule mediating trophoblast dysfunction in a mouse model [64], and Tolllike receptor 4 has been demonstrated to mediate the in vitro inhibition of trophoblast invasion induced by purified aPL IgG from patients with OAPS but not by aPL IgG from non-OAPS[65].

\section{Diagnosis}

\section{Association between aPLs and clinical events}

The association between different obstetrical events and aPLs has been studied with contrasting results that have been critically reviewed by a Task Force comprising international experts during the 14th International Congress on aPLs [66]. In particular, even if the majority of numerous studies report a positive association between aPLs and REPL, they were affected by significant methodological heterogeneity with only very few studies meeting the classification criteria. The experts concluded that few studies support an association between aPLs and REPL. Similarly, FD, the first obstetrical complication associated with aPLs that is considered to be the most specific feature of OAPS, was associated with aPLs in few studies including a systematic literature review [67] and a large prospective population-based study [68]. The literature concerning the relationship between aPLs and PreE and placental insufficiency (PI) is even more tenuous (case-control studies and cohort studies, respectively, in the majority of cases) [66]. A critical review of the literature on an association of aPLs with clinical manifestations found that the differences in the frequencies of aPLs between patients and controls were significant for overall pregnancy morbidity, pregnancy loss, FD, and severe PreE but not for REPL, IUGR, PreE, eclampsia, and HELLP syndrome [69]. In summary, multicenter studies using classification criteria for inclusion are needed to confirm the association between aPLs and obstetrical morbidity [66]. The standardization of aPL tests between centers is critical. Independent laboratories, 
as those created by the APS action network [70], could have a significant role in confirming positive tests.

aPLs, not included in the classification criteria, such as anti-phosphatidylethanolamine, aPT, aPT/PS, anti-phosphatidylinositol (aPI), and anti-A5, have been proposed to play a role in OAPS based on the observation of a higher prevalence in women with pregnancy morbidity [71], [72] and [73]. The Task Force on laboratory diagnostic and trends of the 14th International Congress on aPLs concluded that aPS and aPI may identify additional women with REPL [74], but that there is insufficient evidence to recommend routine testing for non-criteria antibodies at this time.

\section{Risk stratification}

Another matter of debate is the stratification of risk for pregnancy morbidity in aPL-positive patients. Historically, aPL titers have been considered important in this context and patients with high titers have been identified as those with higher risk of events. According to the classification criteria, only women with pregnancy morbidity and medium-high-titer aPLs should be diagnosed with OAPS. A pregnancy outcome similar to healthy controls was previously reported in women with low-titer aPLs supporting this hypothesis [75]. However, there is increasing evidence that such patients can experience poor pregnancy outcomes similarly to high-titer patients [76], [77] and [78]. These observations suggest that, in contrast to thrombotic events, low-titer aPLs can play a significant role in OAPS and that the actual classification criteria do not include all the OAPS cases. Among aPL specificities, LA and triple positivity have been identified as markers of worse outcome[79], $*[80]$ and [69]. Finally, the presence of an associated systemic autoimmune disease, in particular SLE, a history of thrombotic events, and complement reduction have been recognized as being predictive of a poor pregnancy outcome [79] and [81]. Recently, the detection of altered angiogenic biomarkers in combination with clinical criteria during early gestation has been suggested as another tool predictive of adverse pregnancy outcome[82].

\section{Instrumental tools}

Serial obstetrical ultrasonography to assess fetal growth/morphology and placental characteristics is an essential tool to identify pregnancy complications related to aPLs. Furthermore, uterine artery Doppler analysis during the second trimester has been used increasingly as a screening method to detect pregnancy complications associated with uteroplacental insufficiency, before the onset of clinical consequences [83]. Interest in earlier prediction of pregnancy morbidity has led to studies of the role of uterine artery Doppler analysis in the first trimester, which showed good accuracy for predicting early PreE and IUGR, particularly when combined with maternal characteristics and biochemical biomarkers [84]. New techniques including magnetic resonance imaging to detect PI and abnormal fetal brain development are under investigation as noninvasive screening methods to predict placental insufficiency-related complications [85]. 


\section{Treatment}

\section{Standard treatment}

The rate of successful pregnancy outcomes in APS patients without any treatment is very low (20$30 \%$ ), while with current treatment, APS patients have a $70-80 \%$ chance of a live birth [80]. Although controversial, due to a limited number of trials that mostly included patients who did not meet criteria, the current standard of care for pregnant patients fulfilling criteria for APS, based on international recommendations, consists of the following $*[66]$ and $*[86]$.

LDA combined with prophylactic doses of unfractionated heparin or LMWH for patients fulfilling the APS classification criteria based on a history of pregnancy morbidity only;

LDA and therapeutic doses of unfractionated heparin or LMWH (switching from warfarin as soon as pregnancy is confirmed) for patients fulfilling the APS Classification Criteria based on a thrombotic event regardless of a history of pregnancy.

The efficacy of heparin and LDA together in APS patients with previous pregnancy loss is supported by three meta-analyses [87], [88] and [89]. There is less evidence for the same approach in manifestations other than pregnancy loss. Furthermore, the common practice of using LMWH, instead of unfractionated heparin, is not supported by robust evidence, as only unfractionated heparin has shown a beneficial effect when added to LDA, whereas data on LMWH are inconclusive [66]. LDA has been shown not only to prevent placental thrombosis by its well-known anti-platelet effect but also to favor placental development by increasing interleukin-3 [90]. Heparin, beyond its anticoagulant effect, is able to prevent aPL binding to trophoblast, to antagonize the aPL-induced interference with trophoblast syncytialization, apoptosis, invasion, and hormonal production, to antagonize aPL-induced complement activation, to promote the cleavage of $\beta 2 \mathrm{GPI}$, but also to block the aPL-mediated inhibition of endometrial endothelial cell angiogenic differentiation, to aid the clearance of pro-inflammatory chemokines, and to block inflammatory cell adhesion and migration through endothelial cells [59] and [91].

\section{Treatment of refractory cases}

In patients failing LDA and heparin combination, although there are no evidence-based recommendations, common next steps used by physicians with experience in OAPS are as follows: increasing LMWH (from a prophylactic to a therapeutic dose) and/or adding one or more molecules among HCQ, low-dose corticosteroids, IVIg, and/or apheresis [92]. The use of such strategies could improve the obstetrical outcome, but the best regimen is yet to be identified.

The rationale for HCQ is based on its ability, demonstrated in vitro, to reduce aPL binding to the trophoblast and to restore the expression of annexin 5 at the placental level [93]. The addition of HCQ to the standard therapy in refractory OAPS recently showed a reduction in pregnancy loss from $81 \%$ to 
$19 \%$ in a retrospective multicenter study [80]. That result is supported by a retrospective single-center study on aPL-positive patients that showed that HCQ significantly increased the rate of live births (from 57\% vs. 67\%) and reduced the risk of any obstetrical complication [94]. The ability of HCQ to reduce aPL levels has been proposed as an additional mechanism of action in OAPS [95]. High-dose corticosteroids (e.g., prednisolone 40-60 mg/day) are associated with multiple complications during pregnancy (e.g., gestational diabetes, hypertensive disorders, and premature rupture of membranes). Nonetheless, based on the ability of steroids to impair complement activation both in vitro and in vivo [96] and the common practice in recent decades of using steroids in refractory cases, a group demonstrated that low-dose $(10 \mathrm{mg})$ prednisolone during the first trimester was associated with an increased rate of live births in a small cohort [97].

Apheresis (plasma exchange or immunoadsorption), with the aim of removing aPLs from the maternal circulation, has been used in high-risk OAPS with success in some case series [98], [99] and [100] but evidence of a clear benefit is still lacking.

IVIg has also been used in refractory OAPS based on its ability to inhibit the placental transport and to favor the clearance of maternal autoantibodies but also to modulate cytokine and complement activity [100] and [101]. Nevertheless, both a trial and a Cochrane meta-analysis failed to demonstrate any benefit of adding IVIg to standard treatment in improving the obstetrical outcome of OAPS [102] and [103].

\section{Treatment of non-criteria patients}

No consensus exists for the treatment of non-criteria pregnant patients (low-titer aPLs or suggestive obstetrical history not fulfilling criteria), but most physicians, despite the lack of robust evidence, choose to treat these patients during pregnancy based on the presumption that such aPLs may be clinically relevant $[104]$ and $*[66]$. Another matter of controversy is whether to treat asymptomatic aPL-positive patients (no history of vascular or pregnancy morbidity). A recent systematic review of the literature based on 154 pregnancies was not able to demonstrate superiority of prophylactic treatment with LDA over placebo in such aPL carriers [105]. A recent multicenter study of pregnant patients with confirmed aPL positivity showed that aPL carriers, despite prophylaxis in $100 \%$ of cases, had the same rate of adverse pregnancy outcome as OAPS (18\%), but they were treated with combination regimen (LDA plus heparin) compared to OAPS less often [81].

\section{New drugs}

New drugs for the treatment of OAPS have been studied in recent years. For example, TIFI, a synthetic peptide able to compete and to displace $\beta 2$ GPI molecule from the cell surface including the trophoblast, was able to prevent aPL-mediated fetal loss in mice. However, the observation that mice lacking $\beta 2 \mathrm{GPI}$ had an altered early pregnancy process led researchers to focus on molecules able to prevent aPL-mediated effects without changing the expression of $\beta 2 \mathrm{GPI}$ at placental level [106] and [107]. 


\section{Future research agenda}

Despite advances in the understanding of APS in recent years, many areas need to be investigated further. Although it seems imperative to increase the efforts in determining optimal prognostic markers and therapeutic measures to prevent APS complications, difficulties in conducting randomized control trials in the setting of a relatively rare condition still remain. Well-designed longitudinal observational multicenter studies, such as prospective registries, using agreed classification criteria, validated outcome measures, and standardized laboratory tests should be performed to address the remaining questions.

Recently, some large longitudinal studies have provided further insights on some open questions in the field of APS. Among others, investigators from the Predictors of Pregnancy Outcome: Biomarkers in APL Syndrome and SLE (PROMISSE) study group (ClinicalTrials.gov identifier NCT00198068) have shown that timely risk stratification of patients with aPLs is important for effective clinical care and optimal allocation of health-care resources [79] and [82]. PROMISSE is the largest multicenter, multiethnic, and multiracial study to prospectively assess clinical and laboratory predictors of adverse pregnancy outcome in SLE and/or aPL women with inactive or mild/moderate activity at conception. This study provided a basis for identifying high-risk patients for enrollment in future trials, and a rationale for investigating interventions that target pathways upstream (e.g., complement activation or TNF- $\alpha$ release) or downstream of antiangiogenic factors, sufficiently early to prevent pregnancy morbidity in APS.

Given the rarity of OAPS, a homogeneous database in a multicenter European Registry where physicians could enter patient data was developed recently to facilitate and understand several gaps associated with aPL-related obstetric syndromes [80]. This study showed that the characteristics of OAPS differ from those of classical APS, that all laboratory test categories are needed to avoid falsenegative diagnoses, and that, in some cases, complement levels could act as a serological marker. Cervera et al. [108] recently assessed the prevalence of the main causes of morbidity and mortality in APS during a 10-year follow-up period, in one of the longest observational studies available in APS. They showed that patients with APS still develop significant morbidity (thrombotic events appeared in $16.6 \%$ patients during the first five-year period and in $14.4 \%$ during the second five-year period) and mortality (the survival probability at 10 years was $90.7 \%$ ) despite current treatment.

Future studies are needed to confirm the association of aPL with clinical manifestations reported in the classification criteria and to clarify the role of non-criteria aPLs. They may also support the benefit of standard treatment, identify the best approach for refractory and incomplete cases of APS, and further assess the pleiotropic effects of agents such as statins and HCQ whose addition to anticoagulation may lead to better disease control. Finally, they could help to identify novel diagnostic tools, such as antibodies against DI of $\beta 2 \mathrm{GPI}$ or against PS/PT, which may allow more precise risk stratification, leading to a tailored treatment strategy. 


\section{Summary}

There have been many advances in the understanding of APS in recent years, but many areas need to be further investigated in particular the association between autoantibodies and clinical manifestations, the identification of high-risk patients, the best treatment for each patient category, and the role of new therapeutic strategies. Well-designed longitudinal observational multicenter studies, such as prospective registries, using classification criteria, validated outcome measures, and standardized laboratory tests should be performed to answer the remaining questions.

\section{References}

[1] Miyakis S, Lockshin MD, Atsumi T, et al. International consensus statement on an update of the classification criteria for definite antiphospholipid syndrome (APS). J Thromb Haemost 2006;4:295e306.

[2] Merashli M, Noureldine MH, Uthman I, et al. Antiphospholipid syndrome: an update. Eur J Clin Investig 2015;45: 653e62.

[3] Canaud G, Bienaime F, Tabarin F, et al. Inhibition of the mTORC pathway in the antiphospholipid syndrome. N Engl J Med 2014;371:303e12.

[4] Canaud G, Terzi F. Inhibition of the mTORC pathway in the antiphospholipid syndrome. N Engl J Med 2014;371: 1554e5.

[5] Galli M, Luciani D, Bertolini G, et al. Lupus anticoagulants are stronger risk factors for thrombosis than anticardiolipin antibodies in the antiphospholipid syndrome: a systematic review of the literature. Blood 2003;101:1827e32.

[6] Forastiero R, Martinuzzo M, Pombo G, et al. A prospective study of antibodies to beta2-glycoprotein I and prothrombin, and risk of thrombosis. J Thromb Haemost 2005;3:1231e8.

[7] Pengo V, Ruffatti A, Legnani C, et al. Clinical course of high-risk patients diagnosed with antiphospholipid syndrome. J Thromb Haemost 2010;8:237e42.

[8] Sciascia S, Sanna G, Murru V, et al. GAPSS: the global anti-phospholipid syndrome score. Rheumatology (Oxford) 2013; 52:1397e403.

[9] Galli M, Luciani D, Bertolini G, et al. Anti-beta 2-glycoprotein I, antiprothrombin antibodies, and the risk of thrombosis in the antiphospholipid syndrome. Blood 2003;102:2717e23. $144 \mathrm{M}$. Khamashta et al. / Best Practice \& Research Clinical Rheumatology 30 (2016) 133e148

[10] Urbanus RT, Siegerink B, Roest M, et al. Antiphospholipid antibodies and risk of myocardial infarction and ischaemic stroke in young women in the RATIO study: a case-control study. Lancet Neurol 2009;8:998e1005.

[11] Tektonidou MG, Laskari K, Panagiotakos DB, et al. Risk factors for thrombosis and primary thrombosis prevention in patients with systemic lupus erythematosus with or without antiphospholipid antibodies. Arthritis Rheum 2009;61: 29e36. 
*[12] Sciascia S, Murru V, Sanna G, et al. Clinical accuracy for diagnosis of antiphospholipid syndrome in systemic lupus erythematosus: evaluation of 23 possible combinations of antiphospholipid antibody specificities. J Thromb Haemost 2012;10:2512e8.

[13] Giannakopoulos B, Passam F, loannou Y, et al. How we diagnose the antiphospholipid syndrome. Blood 2009;113: 985e94.

[14] Kenet G, Aronis S, Berkun Y, et al. Impact of persistent antiphospholipid antibodies on risk of incident symptomatic thromboembolism in children: a systematic review and meta-analysis. Semin Thromb Hemost 2011;37:802e9.

[15] Garcia D, Akl EA, Carr R, et al. Antiphospholipid antibodies and the risk of recurrence after a first episode of venous thromboembolism: a systematic review. Blood 2013;122:817e24.

[16] Bertolaccini ML, Amengual O, Atsumi T, et al. 'Non-criteria' aPL tests: report of a task force and preconference workshop at the 13th International Congress on Antiphospholipid Antibodies, Galveston, TX, USA. Lupus April 2010; 2011(20):191e205.

[17] Meijide H, Sciascia S, Sanna G, et al. The clinical relevance of IgA anticardiolipin and IgA anti-beta2 glycoprotein I antiphospholipid antibodies: a systematic review. Autoimmun Rev 2013;12:421e5.

[18] Murthy V, Willis R, Romay-Penabad Z, et al. Value of isolated IgA anti-beta2 -glycoprotein I positivity in the diagnosis of the antiphospholipid syndrome. Arthritis Rheum 2013;65:3186e93.

[19] Andreoli L, Fredi M, Nalli C, et al. Clinical significance of IgA anti-cardiolipin and IgA anti-beta2glycoprotein I antibodies. Curr Rheumatol Rep 2013;15:343.

[20] Bertolaccini ML, Atsumi T, Koike T, et al. Antiprothrombin antibodies detected in two different assay systems. Prevalence and clinical significance in systemic lupus erythematosus. Thromb Haemost 2005;93:289e97.

[21] Pengo V, Denas G, Bison E, et al. Prevalence and significance of anti-prothrombin (aPT) antibodies in patients with Lupus Anticoagulant (LA). Thromb Res 2010;126:150e3.

[22] Bertolaccini ML, Atsumi T, Khamashta MA, et al. Autoantibodies to human prothrombin and clinical manifestations in 207 patients with systemic lupus erythematosus. J Rheumatol 1998;25:1104e8.

[23] Sciascia S, Sanna G, Murru V, et al. Anti-prothrombin (aPT) and anti-phosphatidylserine/prothrombin (aPS/PT) antibodies and the risk of thrombosis in the antiphospholipid syndrome. A systematic review. Thromb Haemost 2013:111.

[24] George J, Gilburd B, Hojnik M, et al. Target recognition of beta2-glycoprotein I (beta2GPI)-dependent anticardiolipin antibodies: evidence for involvement of the fourth domain of beta2GPI in antibody binding. J Immunol 1998;160: 3917e23.

[25] Yang CD, Chen SL, Shen N, et al. Detection of anti-recombinant beta 2-glycoprotein 1 and antirecombinant beta 2- glycoprotein 1 fifth-domain antibodies in sera from patients with systemic lupus erythematosus. Rheumatol Int 1998;18:5e10.

*[26] Andreoli L, Chighizola CB, Nalli C, et al. Clinical characterization of antiphospholipid syndrome by detection of IgG antibodies against beta2 -glycoprotein i domain 1 and domain 4/5: ratio of anti-domain 1 to 
anti-domain $4 / 5$ as a useful new biomarker for antiphospholipid syndrome. Arthritis Rheumatol 2015;67:2196e204.

[27] de Laat B, de Groot PG. Autoantibodies directed against domain I of beta2-glycoprotein I. Curr Rheumatol Rep 2011;13: 70e6.

[28] de Laat B, Pengo V, Pabinger I, et al. The association between circulating antibodies against domain I of beta2- glycoprotein I and thrombosis: an international multicenter study. J Thromb Haemost 2009;7:1767e73.

[29] Banzato A, Pozzi N, Frasson R, et al. Antibodies to Domain I of beta(2)Glycoprotein I are in close relation to patients risk categories in Antiphospholipid Syndrome (APS). Thromb Res 2011;128:583e6.

[30] Erkan D, Yazici Y, Peterson MG, et al. A cross-sectional study of clinical thrombotic risk factors and preventive treatments in antiphospholipid syndrome. Rheumatology (Oxford) 2002;41:924e9.

[31] Matyja-Bednarczyk A, Swadzba J, Iwaniec T, et al. Risk factors for arterial thrombosis in antiphospholipid syndrome. Thromb Res 2014;133:173e6.

[32] Esdaile JM, Abrahamowicz M, Grodzicky T, et al. Traditional Framingham risk factors fail to fully account for accelerated atherosclerosis in systemic lupus erythematosus. Arthritis Rheum 2001;44:2331e7.

*[33] Kaiser R, Cleveland CM, Criswell LA. Risk and protective factors for thrombosis in systemic lupus erythematosus: results from a large, multi-ethnic cohort. Ann Rheum Dis 2009;68:238e41.

[34] Lefevre G, Lambert M, Bacri JL, et al. Thrombotic events during long-term follow-up of obstetric antiphospholipid syndrome patients. Lupus 2011;20:861e5.

[35] Gris JC, Bouvier S, Molinari N, et al. Comparative incidence of a first thrombotic event in purely obstetric antiphospholipid syndrome with pregnancy loss: the NOH-APS observational study. Blood 2012;119:2624e32.

[36] Martinez-Zamora MA, Peralta S, Creus M, et al. Risk of thromboembolic events after recurrent spontaneous abortion in antiphospholipid syndrome: a case-control study. Ann Rheum Dis 2012;71:61e6.

[37] Andreoli L, Tincani A. Beyond the "syndrome": antiphospholipid antibodies as risk factors. Arthritis Rheum 2012;64: 342e5.

[38] Otomo K, Atsumi T, Amengual O, et al. Efficacy of the antiphospholipid score for the diagnosis of antiphospholipid syndrome and its predictive value for thrombotic events. Arthritis Rheum 2012;64:504e12.

[39] Sciascia S, Cosseddu D, Montaruli B, et al. Risk Scale for the diagnosis of antiphospholipid syndrome. Ann Rheum Dis 2011;70:1517e8.

[40] Sciascia S, Cuadrado MJ, Sanna G, et al. Thrombotic risk assessment in systemic lupus erythematosus: validation of the global antiphospholipid syndrome score in a prospective cohort. Arthritis Care Res Hob 2014;66:1915e20.

[41] Sciascia S, Sanna G, Murru V, et al. The global anti-phospholipid syndrome score in primary APS. Rheumatology (Oxford) 2015;54:134e8. M. Khamashta et al. / Best Practice \& Research Clinical Rheumatology 30 (2016) 133e148 145

[42] Zuily S, de Laat B, Mohamed S, et al. Validity of the global anti-phospholipid syndrome score to predict thrombosis: a prospective multicentre cohort study. Rheumatology (Oxford) 2015;54:2071e5. 
*[43] Erkan D, Aguiar CL, Andrade D, et al. 14th International Congress on antiphospholipid antibodies: task force report on antiphospholipid syndrome treatment trends. Autoimmun Rev 2014;13:685e96.

[44] Ginsburg KS, Liang MH, Newcomer L, et al. Anticardiolipin antibodies and the risk for Ischemic stroke and venous thrombosis. Ann Intern Med 1992;117:997e1002.

[45] Becattini C, Agnelli G, Schenone A, et al. Aspirin for preventing the recurrence of venous thromboembolism. N. Engl J Med 2012;366:1959e67.

[46] Cuadrado MJ, Bertolaccini ML, Seed PT, et al. Low-dose aspirin vs low-dose aspirin plus low-intensity warfarin in thromboprophylaxis: a prospective, multicentre, randomized, open, controlled trial in patients positive for antiphospholipid antibodies (ALIWAPAS). Rheumatology (Oxford) 2014;53:275e84.

[47] Kearon C, Ginsberg JS, Kovacs MJ, et al. Comparison of low-intensity warfarin therapy with conventionalintensity warfarin therapy for long-term prevention of recurrent venous thromboembolism. $\mathrm{N}$ Engl J Med 2003;349:631e9.

[48] Finazzi G, Marchioli R, Brancaccio V, et al. A randomized clinical trial of high-intensity warfarin vs. conventional antithrombotic therapy for the prevention of recurrent thrombosis in patients with the antiphospholipid syndrome (WAPS). J Thromb Haemost 2005;3:848e53.

[49] Pengo V, Ruiz-Irastorza G, Denas G, et al. High intensity anticoagulation in the prevention of the recurrence of arterial thrombosis in antiphospholipid syndrome: 'PROS' and 'CONS'. Autoimmun Rev 2012;11:577e80.

[50] Levine SR, Brey RL, Tilley BC, et al. Antiphospholipid antibodies and subsequent thrombo-occlusive events in patients with ischemic stroke. JAMA 2004;291. 576e584.

*[51] Ruiz-Irastorza G, Hunt BJ, Khamashta MA. A systematic review of secondary thromboprophylaxis in patients with antiphospholipid antibodies. Arthritis Rheum 2007;57:1487e95.

[52] Ruiz-Irastorza G, Khamashta MA, Hunt BJ, et al. Bleeding and recurrent thrombosis in definite antiphospholipid syndrome: analysis of a series of 66 patients treated with oral anticoagulation to a target international normalized ratio of 3.5. Archives Intern Med 2002;162:1164e9.

[53] Sciascia S, Lopez-Pedrera C, Cecchi I, et al. Non-vitamin K antagonist oral anticoagulants and antiphospholipid syndrome. Rheumatology (Oxford) 2016 Feb 3. pii: kev445. [Epub ahead of print].

[54] Sciascia S, Khamashta MA, D'Cruz DP. Targeted therapy in antiphospholipid syndrome. Curr Opin Rheumatol 2014;26: 269e75.

[55] Erkan D, Vega J, Ramon G, et al. A pilot open-label phase II trial of rituximab for non-criteria manifestations of antiphospholipid syndrome. Arthritis Rheum 2013;65:464e71.

[56] Bakimer R, Fishman P, Blank M, et al. Induction of primary antiphospholipid syndrome in mice by immunization with a human monoclonal anticardiolipin antibody (H-3). J Clin Investig 1992;89:1558e63.

[57] Rand JH, Wu XX, Quinn AS, et al. The annexin A5-mediated pathogenic mechanism in the antiphospholipid syndrome: role in pregnancy losses and thrombosis. Lupus 2010;19:460e9. 
[58] Salafia CM, Cowchock FS. Placental pathology and antiphospholipid antibodies: a descriptive study. Am J Perinatol 1997;14:435e41.

[59] Tong M, Viall CA, Chamley LW. Antiphospholipid antibodies and the placenta: a systematic review of their in vitro effects and modulation by treatment. Hum Reprod Update 2015;21:97e118.

[60] Di Simone N, Meroni PL, de Papa N, et al. Antiphospholipid antibodies affect trophoblast gonadotropin secretion and invasiveness by binding directly and through adhered beta2-glycoprotein I. Arthritis Rheum 2000;43:140e50.

[61] Viall CA, Chen Q, Liu B, et al. Antiphospholipid antibodies internalised by human syncytiotrophoblast cause aberrant cell death and the release of necrotic trophoblast debris. J Autoimmun 2013;47:45e7.

*[62] Meroni PL, Borghi MO, Raschi E, et al. Pathogenesis of antiphospholipid syndrome: understanding the antibodies. Nat Rev Rheumatol 2011;7:330e9.

[63] Müller-Calleja N, Kohler A, Siebald B, et al. Cofactor-independent antiphospholipid antibodies activate the NLRP3- $€$ inflammasome via endosomal NADPH-oxidase: implications for the antiphospholipid syndrome. Thromb Haemost 2015;113:1071e83.

[64] Ulrich V, Gelber SE, Vukelic M, et al. ApoE Receptor 2 mediates trophoblast dysfunction and pregnancy complications induced by antiphospholipid antibodies in mice. Arthritis Rheumatol 2016;68:730e9.

[65] Poulton K, Ripoll VM, Pericleous C, et al. Purified IgG from patients with obstetric but not IgG from nonobstetric antiphospholipid syndrome inhibit trophoblast invasion. Am J Reprod Immunol 2015;73:390e401. *[66] Ramires de Jesus G, Agmon-Levin N, Andrade CA, et al. 14th International congress on antiphospholipid antibodies task force report on obstetric antiphospholipid syndrome. Autoimmun Rev 2014;13:795e813.

[67] Abou-Nassar K, Carrier M, Ramsay T, et al. The association between antiphospholipid antibodies and placenta mediated complications: a systematic review and meta-analysis. Thromb Res 2011;128:77e85.

[68] Silver RM, Parker CB, Reddy UM, et al. Antiphospholipid antibodies in stillbirth. Obstet Gynecol 2013;122:641e57.

[69] Chighizola CB, Andreoli L, Ramires de Jesus G, et al. The association between antiphospholipid antibodies and pregnancy morbidity, stroke, myocardial infarction, and deep vein thrombosis : a critical review of the literature. Lupus 2015;24:980e4.

[70] Erkan D, Lockshin MD, APS ACTION members. APS ACTION-AntiPhospholipid syndrome Alliance for clinical trials and InternatiOnal networking. Lupus 2012;21:695e8.

[71] Ulcova-Gallova Z, Krauz V, Novakova P, et al. Anti-phospholipid antibodies against phosphatidylinositol, and phosphatidylserine are more significant in reproductive failure than antibodies against cardiolipin only. Am J Reprod Immunol 2005;54:112e7.

[72] Zigon P, Perdan Pirkmajer K, Tomsic M, et al. Anti-Phosphatidylserine/Prothrombin antibodies are associated with adverse pregnancy outcomes. J Immunol Res 2015;2015:975704.

[73] Sater MS, Finan RR, Abu-Hijleh FM, et al. Anti-phosphatidylserine, anti-cardiolipin, anti-b2 glycoprotein I and antiprothrombin antibodies in recurrent miscarriage at 8-12 gestational weeks. Eur J Obstet Gynecol 
Reprod Biol 2012; 163:170e4. 146 M. Khamashta et al. / Best Practice \& Research Clinical Rheumatology 30 (2016) $133 \mathrm{e} 148$

[74] Bertolaccini ML, Amengual O, Andreoli L, et al. 14th International congress on antiphospholipid antibodies task force. Report on antiphospholipid syndrome laboratory diagnostics and trends. Autoimmun Rev 2014;13:917e30.

[75] Simchen MJ, Dulitzki M, Rofe G, et al. High positive antibody titers and adverse pregnancy outcome in women with antiphospholipid syndrome. Acta Obstet Gynecol Scand 2011;90:1428e33.

[76] Boffa MC, Boinot C, De Carolis S, et al. Laboratory criteria of the obstetrical antiphospholipid syndrome. Data from a multicentric prospective European women cohort. Thromb Haemost 2009;102:25e8.

[77] Gardiner C, Hills J, Machin SJ, et al. Diagnosis of antiphospholipid syndrome in routine clinical practice. Lupus 2013; 22:18e25.

[78] Ofer-Shiber S, Molad Y. Frequency of vascular and pregnancy morbidity in patients with low vs. moderateto-high titers of antiphospholipid antibodies. Blood Coagul Fibrinolysis 2015;26:261e6.

[79] Lockshin MD, Kim M, Laskin CA, et al. Prediction of adverse pregnancy outcome by the presence of lupus anticoagulant, but not anticardiolipin antibody, in patients with antiphospholipid antibodies. Arthritis Rheum 2012;64: 2311e8.

*[80] Alijotas-Reig J, Ferrer-Oliveras R, Ruffatti A, et al. The European registry on obstetric antiphospholipid syndrome (EUROAPS): a survey of 247 consecutive cases. Autoimmun Rev 2015;14:387e95.

[81] Fredi M, Aggogeri E, Bettiga E, et al. A multicenter evaluation of obstetric and maternal outcome in prospectively followed pregnant patients with confirmed positivity for antiphospholipid antibodies (apl) [abstract]. Arthritis Rheumatol 2015;67(Suppl. 10).

[82] Kim MY, Buyon JP, Guerra MM, et al. Angiogenic factor imbalance early in pregnancy predicts adverse outcomes in patients with lupus and antiphospholipid antibodies: results of the PROMISSE study. Am J Obstet Gynecol 2016;214: 108.e1e108.e14.

[83] Cnossen JS, Morris RK, ter Riet G, et al. Use of uterine artery Doppler ultrasonography to predict preeclampsia and intrauterine growth restriction: a systematic review and bivariable meta-analysis. CMAJ 2008;178:701e11.

[84] Velauthar L, Plana MN, Kalidindi M, et al. First-trimester uterine artery Doppler and adverse pregnancy outcome: a meta-analysis involving 55,974 women. Ultrasound Obstet Gynecol 2014;43:500e7.

[85] Girardi G. MRI-based methods to detect placental and fetal brain abnormalities in utero. J Reprod Immunol 2016;114: 86e91.

*[86] Bates SM, Greer IA, Middeldorp S, et al. American college of chest physicians. VTE, thrombophilia, antithrombotic therapy, and pregnancy: antithrombotic therapy and prevention of thrombosis, 9th ed: American college of chest physicians evidence-based clinical practice guidelines. Chest 2012;141:e691Se736S.

[87] Empson M, Lassere M, Craig J, et al. Prevention of recurrent miscarriage for women with antiphospholipid antibody or lupus anticoagulant. Cochrane Database Syst Rev 2005;18. CD002859. 
[88] Mak A, Cheung MW, Cheak AA, et al. Combination of heparin and aspirin is superior to aspirin alone in enhancing live births in patients with recurrent pregnancy loss and positive anti-phospholipid antibodies: a meta-analysis of randomized controlled trials and meta-regression. Rheumatology (Oxford) 2010;49:281e8.

[89] Ziakas PD, Pavlou M, Voulgarelis M. Heparin treatment in antiphospholipid syndrome with recurrent pregnancy loss: a systematic review and meta-analysis. Obstet Gynecol 2010;115:1256e12562.

[90] Fishman P, Falach-Vaknin E, Sredni B, et al. Aspirin-interleukin-3 interrelationships in patients with antiphospholipid syndrome. Am J Reprod Immunol 1996;35:80e4.

[91] Mastrolia SA, Mazor M, Holcberg G, et al. The physiologic anticoagulant and anti-inflammatory role of heparins and their utility in the prevention of pregnancy complications. Thromb Haemost 2015;113:1236e46.

[92] Gomez-Puerta JA, Cervera R. Are there additional options for the treatment of refractory obstetric antiphospholipid syndrome? Lupus 2013;22:754e5.

[93] Wu XX, Guller S, Rand JH. Hydroxychloroquine reduces binding of antiphospholipid antibodies to syncytiotrophoblasts and restores annexin A5 expression. Am J Obstet Gynecol 2011;205. 576e514.

[94] Siascia S, Hunt BJ, Talaver-Garcia E, et al. The impact of hydroxychloroquine treatment on pregnancy outcome in women with antiphospholipid antibodies. Am J Obstet Gynecol 2016;214:273.e1e8.

[95] De Carolis S, Botta A, Salvi S, et al. Is there any role for the hydroxychloroquine (HCQ) in refractory obstetrical antiphospholipid syndrome (APS) treatment. Autoimmun Rev 2015;14:760e2.

[96] Sneiderman C, Wilson J. Effects of corticosteroids on complement and the neutrophilic polymorphonuclear leukocyte. Transpl Proc 1975;7:41e8.

[97] Bramham K, Thomas M, Nelson-Piercy C, et al. First-trimester low-dose prednisolone in refractory antiphospholipid antibody-related pregnancy loss. Blood 2011;117:6948e51.

[98] Ruffatti A, Marson P, pengo V, et al. Plasma exchange in the management of high risk pregnant patients with primary antiphospholipid syndrome. A case report of 9 cases and a review of the literature. Autoimmun Rev 2007;6:196e202.

[99] Bortolati M, Marson P, Chairelli S, et al. Case reports of the use of immunoadsorption or plasma exchange in high-risk pregnancies of women with antiphospholipid syndrome. Ther Apher Dial 2009;13:157e60.

[100] Ruffatti A, Favaro M, Brucato A, et al. Apheresis in high risk antiphospholipid syndrome pregnancy and autoimmune congenital heart block. Transfus Apher Sci 2015;53:269e78.

[101] Yu Z, Lennon VA. Mechanism of intravenous immune globulin therapy in antibody-mediated autoimmune diseases. N Engl J Med 1993;340:227e8.

[102] Dendrinos S, Sakkas E, Makrakis E. Low-molecular-weight heparin versus intravenous immunoglobulin for recurrent abortion associated with antiphospholipid antibody syndrome. Int J Gynaecol Obstet 2009;104:223e5.

[103] Empson M, Lassere M, Craig J, et al. Prevention of recurrent miscarriage for women with antiphospholipid antibody or lupus anticoagulant. Cochrane Database Syst Rev 2005;2. CD002859. 
[104] Arachchillage DRJ, Machin SJ, Mackie IJ, et al. Diagnosis and management of non-criteria obstetric antiphospholipid syndrome. Thromb Haemost 2015;113:13e9.

[105] Amengual O, Fujita D, Carmona L, et al. Primary prophylaxis to prevent obstetric complications in asymptomatic women with antiphospholipid antibodies: a systematic review. Lupus 2015;24:1135e42. M. Khamashta et al. / Best Practice \& Research Clinical Rheumatology 30 (2016) 133e148 147

[106] De la Torre YM, Pregnolato F, D'Amelio F, et al. Anti-phospholipid induced murine fetal loss: novel protective effect of a peptide targeting the b2 glycoprotein I phospholipid-binding site. Implications for human fetal loss. J Autoimmun 2012;38:J209e15.

[107] Agostinis C, Durigutto $P$, Sblattero D, et al. A non-complement-fixing antibody to b2 glycoprotein I as a novel therapy for antiphospholipid syndrome. Blood 2014;123:3478e87.

[108] Cervera R, Serrano R, Pons-Estel GJ, et al. Euro-Phospholipid Project Group (European Forum on Antiphospholipid Antibodies). Morbidity and mortality in the antiphospholipid syndrome during a 10-year period: a multicentre prospective study of 1000 patients. Ann Rheum Dis 2015;74. 1011e1008. 
Table 1. Revised classification criteria for antiphospholipid syndrome.

Vascular $\quad \geq 1$ clinical episode of arterial, venous, or small vessel thrombosis. Thrombosis must be objectively thrombosis confirmed. For histopathological confirmation, thrombosis must be present without inflammation of the vessel wall

Pregnancy morbidity

Laboratory criteria

$$
\geq 1 \text { unexplained death of a morphologically normal fetus } \geq 10 \text { weeks of gestation }
$$

2

$\geq 1$ premature delivery of a morphologically normal fetus $<34$ weeks of gestation because of:

severe preeclampsia or eclampsia defined according to standard definition;

recognized features of placental insufficiency

3

$\geq 3$ unexplained consecutive miscarriages $<10$ weeks of gestation, with maternal and paternal factors (anatomic, hormonal, or chromosomal abnormalities) excluded

Presence of antiphospholipid antibodies (aPL), on two or more occasions at least 12 weeks apart and no more than 5 years prior to clinical manifestations, as demonstrated by $\geq 1$ of the following:

a Lupus anticoagulant;

b Medium to high-titer ( $>40$ GPL or MPL, or $>99$ th percentile) anticardiolipin IgG or IgM;

c Anti- $\beta 2$ glycoprotein-I (anti- $\beta 2$ GPI) IgG or IgM $>99$ th percentile

Table 2. Antiphospholipid antibody (aPL) risk categories according to profile.

High-risk aPL profile
Lupus anticoagulant positivity

Triple positivity (lupus anticoagulant + anti-cardiolipin + anti- $\beta 2$ glycoprotein-I antibodies)

Isolated persistently positive anti-cardiolipin antibodies at medium-high titers

Low-risk aPL profile
Isolated, intermittently positive anti-cardiolipin or anti- $\beta 2$ glycoprotein- I at low-medium titers 
Table 3. The global antiphospholipid syndrome score (GAPSS).

\section{Factor}

Anticardiolipin IgG/IgM

Anti- $\beta 2$-glycoprotein IgG/IgM

Lupus anticoagulant

Anti-prothrombin/phosphatidylserine complex (aPS/PT) IgG/IgM

Hyperlipidemia

Arterial hypertension

\section{Point value}

5

4

4

3

3

1

The GAPSS scoring system is derived from the combination of independent risk for both thrombosis and pregnancy loss, and accounted for multiple factors, including the patient's aPL profile, conventional cardiovascular risk factors, autoimmune antibody profile, and thromboprophylactic drug use. The GAPSS can be calculated for each patient by adding the points corresponding to the different risk factors, weighted as shown. GAPSS values $\geq 10$ have demonstrated the best diagnostic accuracy compared with the different thresholds for APS diagnosis. 